\title{
Impaired Efflux of the Siderophore Enterobactin Induces Envelope Stress in Escherichia coli
}

\author{
Randi L. Guest ${ }^{\dagger}$, Emily A. Court, Jayne L. Waldon, Kiersten A. Schock and Tracy L. Raivio* \\ Department of Biological Sciences, University of Alberta, Edmonton, AB, Canada
}

OPEN ACCESS

Edited by:

Haike Antelmann,

Freie Universität Berlin,

Germany

Reviewed by:

Kunihiko Nishino,

Osaka University,

Japan

Jon William Weeks,

United States Food and Drug

Administration, United States

${ }^{*}$ Correspondence:

Tracy L. Raivio

traivio@ualberta.ca

tPresent address:

Randi L. Guest,

Lewis Thomas Laboratory,

Department of Molecular Biology,

Princeton University, Princeton,

NJ, United States

Specialty section:

This article was submitted to

Microbial Physiology and Metabolism,

a section of the journal

Frontiers in Microbiology

Received: 30 September 2019 Accepted: 14 November 2019

Published: 06 December 2019

Citation:

Guest RL, Court EA, Waldon JL, Schock KA and Raivio TL (2019)

Impaired Efflux of the Siderophore

Enterobactin Induces Envelope

Stress in Escherichia coli.

Front. Microbiol. 10:2776.

doi: 10.3389/fmicb.2019.02776
The Cpx response is one of several envelope stress responses that monitor and maintain the integrity of the gram-negative bacterial envelope. While several conditions that are known or predicted to generate misfolded inner membrane proteins activate the Cpx response, the molecular nature of the Cpx inducing cue is not yet known. Studies have demonstrated that mutation of multidrug efflux pumps activates the Cpx response in many gram-negative bacteria. In Vibrio cholerae, pathway activation is due to accumulation of the catechol siderophore vibriobactin. However, the mechanism by which the Cpx response is activated by mutation of efflux pumps in Escherichia coli remains unknown. Here we show that inhibition of efflux by deletion of to/C, the outer membrane channel of several multidrug efflux pumps, activates the Cpx response in $E$. coli as a result of impaired efflux of the siderophore enterobactin. Enterobactin accumulation in the tolC mutant reduces activity of the nicotinamide adenine dinucleotide (NADH) oxidation arm of the aerobic respiratory chain. However, the $\mathrm{Cpx}$ pathway remains active in the to/C mutant when either $\mathrm{NADH}$ dehydrogenase I, $\mathrm{NADH}$ dehydrogenase $\mathrm{I}$, or cytochrome $b_{3}$ is absent. Finally, we show that the Cpx response down-regulates transcription of the enterobactin biosynthesis operon. These results suggest that the Cpx response promotes adaptation to envelope stress in enteric bacteria that are exposed to iron-limited environments, which are rich in envelope-damaging compounds and conditions.

Keywords: Cpx stress response, TolC, efflux, enterobactin, respiration, electron transport chain

\section{INTRODUCTION}

In order for antimicrobial compounds to gain access to their cellular target, they must first cross one or more layers of the bacterial envelope. In gram-negative bacteria, this envelope consists of the outer membrane, the inner membrane, and the peptidoglycan sacculus located within the intervening periplasmic space (Silhavy et al., 2010). Antimicrobials that have crossed the envelope may be transported out of the cell via multidrug efflux pumps. Some multidrug efflux pumps interact with an outer membrane channel and periplasmic membrane fusion protein to form a tripartite protein complex that can directly transport toxic molecules from the cytoplasm or periplasm to the external environment, while others function as single component pumps that transport compounds from the cytoplasm to the periplasm ( $\mathrm{Li}$ et al., 2015). Compounds transported to the periplasm via singlet efflux pumps may then move out of the cell through a tripartite machine (Lee et al., 2000; Tal and Schuldiner, 2009).

Escherichia coli encode several tripartite multidrug efflux systems, many of which use the same outer membrane channel, TolC (Li et al., 2015). Decades of research have shown that TolC is 
required for the efflux of a wide variety of dyes, detergents, and antibiotics. However, there is a growing body of evidence to suggest that TolC is also required for the secretion of endogenously produced metabolites. Intra- and extracellular concentrations of cysteine, indole, porphyrins, and siderophores are affected by loss of TolC or TolC-dependent efflux pumps (Bleuel et al., 2005; Tatsumi and Wachi, 2008; Wiriyathanawudhiwong et al., 2009; Horiyama and Nishino, 2014). Furthermore, accumulation of several metabolites increases expression of the TolC-dependent AcrAB multidrug efflux system as a compensatory mechanism to increase metabolite secretion (Helling et al., 2002; Ruiz and Levy, 2014). Blocking metabolite secretion by mutating tolC or TolC-dependent efflux systems increases sensitivity to cysteine, the siderophore enterobactin, and intermediates of heme biosynthesis, suggesting that metabolite accumulation is toxic (Tatsumi and Wachi, 2008; Wiriyathanawudhiwong et al., 2009; Vega and Young, 2014). In support of this hypothesis, numerous cellular stress responses are activated in bacteria lacking tolC (Rosner and Martin, 2009; Guest and Raivio, 2016a), including the Cpx envelope stress response.

Current evidence suggests that the Cpx envelope stress response functions to monitor and maintain the biogenesis of inner membrane proteins and protein complexes (Vogt and Raivio, 2012; Raivio, 2014; Guest et al., 2017). This response is controlled by a typical two-component signal transduction system consisting of the inner membrane-bound sensor CpxA and the cytoplasmic response regulator CpxR (Weber and Silverman, 1988; Dong et al., 1993). In the presence of an inducing signal, CpxA autophosphorylates and the phosphate is then transferred to CpxR (Raivio and Silhavy, 1997). Once phosphorylated, CpxR functions as a transcription factor to activate the expression of genes associated with protein biogenesis and inner membrane integrity (Danese et al., 1995; Danese and Silhavy, 1997, 1998; Pogliano et al., 1997; Raivio et al., 2000, 2013; Price and Raivio, 2009), and repress the expression of genes that encode macromolecular envelope-localized protein complexes (McEwen and Silverman, 1980; Dorel et al., 1999; Hernday et al., 2004; MacRitchie et al., 2008; Vogt et al., 2010; Acosta et al., 2015; Guest et al., 2017). Once homeostasis is achieved, CpxA functions as a phosphatase to dephosphorylate CpxR and attenuate the response (Raivio and Silhavy, 1997).

Inhibition of efflux activates the Cpx response in several gram-negative bacteria, including E. coli, Vibrio cholerae, Sinorhizobium meliloti, and Haemophilus ducreyi (Slamti and Waldor, 2009; Santos et al., 2010; Rinker et al., 2011; Rosner and Martin, 2013; Acosta et al., 2014; Taylor et al., 2014), and is the most conserved Cpx-inducing cue identified to date. Clues as to how impaired efflux activates the Cpx response have come from studies in $V$. cholerae. Activation of the Cpx pathway in $V$. cholerae lacking the TolC-dependent efflux system VexGH is suppressed when $V$. cholerae are grown in the presence of iron, suggesting that the metabolite responsible for activation of the $V$. cholerae Cpx response is produced when iron is limiting (Acosta et al., 2014). In a subsequent study, this metabolite was identified as the catechol siderophore vibriobactin (Kunkle et al., 2017). This study also found that the V. cholerae Cpx response is no longer activated in an efflux mutant when bacteria are grown anaerobically or when succinate dehydrogenase of the electron transport chain is disrupted. As such, it has been proposed that accumulation of vibriobactin activates the Cpx response via the electron transport chain.

It is thought that vibriobactin production is limited to a small number of Vibrio species (Wyckoff et al., 2001; Thode et al., 2018). As such, the mechanism by which inhibition of efflux activates the Cpx response in E. coli remains to be determined. In this study, we show that the catechol siderophore enterobactin is required for activation of the Cpx response in E. coli lacking tolC, suggesting that envelope damage inflicted by impaired secretion of siderophores is a conserved Cpx-inducing signal. While enterobactin was found to decrease activity of the nicotinamide adenine dinucleotide (NADH) oxidation arm of the aerobic electron transport chain in the tolC mutant, loss of $\mathrm{NADH}$ dehydrogenase I, NADH dehydrogenase II, or cytochrome $b_{3}$ does not alter Cpx pathway activity in this background. Finally, we provide evidence to suggest that activation of the Cpx response facilitates adaptation to toxic envelope stresses, such as enterobactin accumulation, by down-regulating the transcription of genes involved in enterobactin biosynthesis.

\section{EXPERIMENTAL PROCEDURES}

\section{Bacterial Strains and Growth Conditions}

All bacterial strains and plasmids used in the course of this study are listed in Supplementary Table S1. Bacteria were grown in either Lennox broth $[\mathrm{LB}, 10 \mathrm{~g} / \mathrm{L}$ Bacto tryptone (Difco), $5 \mathrm{~g} / \mathrm{L}$ yeast extract (Difco), and $5 \mathrm{~g} / \mathrm{L} \mathrm{NaCl}$ ] or $\mathrm{M} 9$ minimal medium (Difco) containing $0.4 \%$ glucose at $37^{\circ} \mathrm{C}$ with shaking at $225 \mathrm{rpm}$. Bacteria were grown at $30^{\circ} \mathrm{C}$ for experiments that included strain TR10 or ALN195. Antibiotics were added as necessary to the following concentrations: amikacin (Amk), $3 \mu \mathrm{g} \mathrm{ml}^{-1}$; ampicillin (Amp), $100 \mu \mathrm{g} \mathrm{ml}^{-1}$; kanamycin (Kan), $50 \mu \mathrm{g} \mathrm{ml}{ }^{-1}$; spectinomycin (Spc), $25 \mu \mathrm{g} \mathrm{ml}{ }^{-1}$; streptomycin (Str), $50 \mu \mathrm{g} \mathrm{ml}^{-1}$. All chemicals were purchased from SigmaAldrich unless otherwise stated.

\section{Strain and Plasmid Construction}

Strains EC3, EC4, RG244, RG249, and RG250 were constructed by P1 transduction (Silhavy et al., 1984). Donor strains, in which the tolC, ent $C, n d h$, or $c p x A$ open-reading frame was replaced with the kanamycin resistance cassette, were obtained from the Keio library (Baba et al., 2006). The kanamycin resistance cassette in the tolC gene was removed by FLP/ FRT-mediated recombination to produce an in-frame, markerless deletion as described in Hoang et al. (1998). All mutations were confirmed by PCR.

Deletion of the nuoABCDEFGHIJKLMN and the cyoABCDE operons in DY378 was performed by lamba-red recombinase as previously described (Thomason et al., 2007). Primer sequences were obtained from Baba et al. (2006) and are listed in Supplementary Table S2. The DNA sequence of the K12nuoKOF primer corresponds to the $5^{\prime}$ primer used to delete nuoA in Baba et al. (2006), while the sequence of the K12nuoKOR primer corresponds to the $3^{\prime}$ primer used to delete nuoN (Baba et al., 
2006). Primer K12-cyoKOF corresponds to the $5^{\prime}$ primer used to delete cyoA in Baba et al. (2006), and primer K12-cyoKOR corresponds to the $3^{\prime}$ primer used to delete $c y o E$ (Baba et al., 2006). PCR was performed using high-fidelity Phusion DNA polymerase (ThermoFisher) according to the manufacturer's specifications with the addition of $20 \%$ betaine. K12nuoKOF and K12nuoKOR, or K12-cyoKOF and K12-cyoKOR, were used to amplify the FRT-flanked kanamycin resistance cassette from the Keio library (Baba et al., 2006). DNA was separated by electrophoresis on a $1 \%$ agarose gel. A DNA fragment approximately the size of the kanamycin resistance cassette was extracted and cleaned using the GeneJet gel purification kit (Fermentas). These DNA fragments were used to delete the nuoA-N or cyoA-E locus in $E$. coli strain DY378, which encodes the lambda-red recombinase system from a temperature-sensitive promoter (Yu et al., 2000; Thomason et al., 2007). Briefly, DY378 was grown to an $\mathrm{OD}_{600}$ of $0.4-0.5$ in $35 \mathrm{ml}$ of $\mathrm{LB}$ in a $250-\mathrm{ml}$ Erlenmeyer flask at $30^{\circ} \mathrm{C}$ with shaking at $225 \mathrm{rpm}$. Half of this culture was then transferred to a $125-\mathrm{ml}$ flask and incubated in a $42^{\circ} \mathrm{C}$ shaking water bath for $15 \mathrm{~min}$, while the other half was incubated at $30^{\circ} \mathrm{C}$ as a control to ensure that kanamycin resistant recombinants obtained in future steps were dependent on the expression of the lambda red recombinase. Cells were washed three times in sequentially lower volumes of ice-cold distilled water, terminating with cells resuspended in 200- $\mu$ l ice-cold distilled water. About 100 or $300 \mathrm{ng}$ of purified kanamycin resistance cassette DNA with homologous ends to the nuo or cyo operon was electroportated into DY378 and cells were recovered at $30^{\circ} \mathrm{C}$ with shaking for $2 \mathrm{~h}$. Recombinants were selected for on LB agar supplemented with kanamycin. Presence of the kanamycin resistance cassette was confirmed by PCR. The nuoA-N::kan and cyoA-E::kan alleles were then moved into strain TR50 by P1 transduction as previously described (Silhavy et al., 1984).

Luminescent transcriptional reporters of entCEBA expression were constructed as previously described (Wong et al., 2013). Briefly, the promoter region of the entCEBA operon was amplified from E2348/69 or MC4100 using the primers PentCluxF and PentCluxR (Supplementary Table S2) and the high-fidelity Phusion DNA polymerase (ThermoFisher) according to the manufacturer's protocol with the addition of $10 \%$ betaine. DNA was separated by electrophoresis on a $1 \%$ agarose gel. DNA bands corresponding to the size of the MC4100 entCEBA promoter and the E2348/69 entCEBA promoter were gel-purified using the GeneJet Gel Purification kit (Fermentas), digested with BamHI and EcoRI (Invitrogen), and ligated upstream of the luxABCDE operon in the pJW15 plasmid. PCR and DNA sequencing verified correct insertion of the promoter sequences. To ensure that the reporters reflect accurate expression of the entCEBA operon, luminescence was determined under ironreplete and iron-deplete conditions. In accordance with published observations, luminescence was reduced in the presence of iron (data not shown). DNA sequencing was performed by the University of Alberta Molecular Biology Services Unit.

\section{$\beta$-Galactosidase Assay}

For Figures 1 and 2, bacteria were grown overnight in LB at $37^{\circ} \mathrm{C}$ with shaking at $225 \mathrm{rpm}$. The following day, strains were
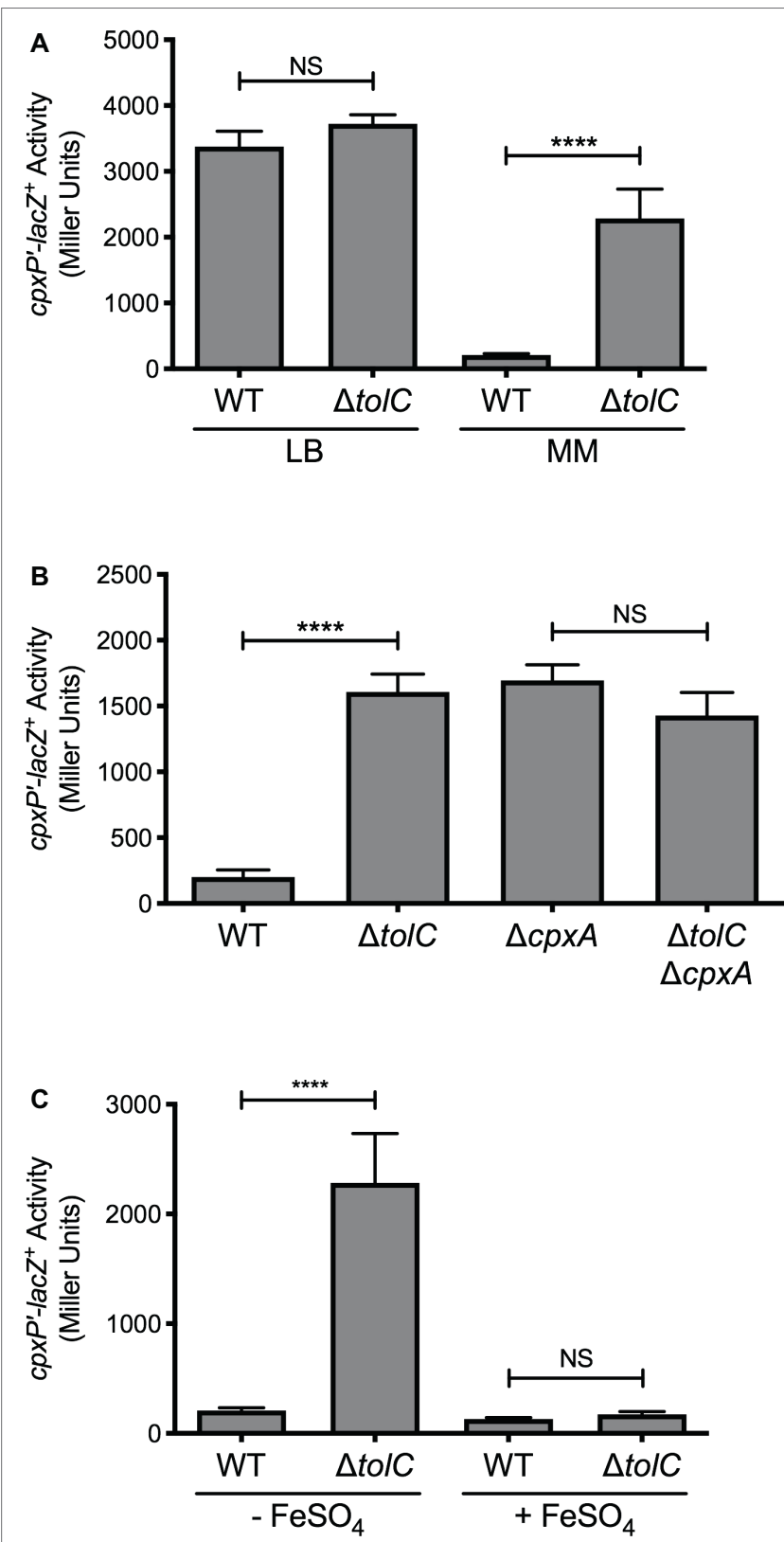

FIGURE 1 | Deletion of tolC activates the E. coli Cpx response under iron-deplete conditions. (A) Wildtype and to/C mutant E. coli MC4100 strains carrying the chromosomal cpxP-lacZ transcriptional reporter were subcultured into Lennox broth (LB) or M9 minimal medium (MM) after overnight growth in $\mathrm{LB}$ medium and grown for $20 \mathrm{~h}$ at $37^{\circ} \mathrm{C}$. (B) cpxP-lacZ activity in wildtype $E$. coli strain MC4100, the to/C and cpxA single mutants, and the to/C cpxA double mutant, grown in M9 minimal medium. (C) Expression of the cpxPlac $Z$ in wildtype and to/C mutant $E$. coli MC4100 strains subcultured into M9 minimal medium with (+) or without (-) $80 \mu \mathrm{M} \mathrm{FeSO}_{4}$ from overnight cultures grown in LB medium. Bacteria were grown for $20 \mathrm{~h}$ at $37^{\circ} \mathrm{C}$. To measure cpxP-lacZ expression, cells were lysed with chloroform and SDS, and $\beta$-galactosidase levels were measured after addition of ONPG in a 96-well plate as described in the experimental procedures. Data represent the means and standard deviations of three biological replicates. Asterisks indicate a statistically significant difference from the indicated wildtype control ${ }^{{ }^{\star \star \star *} p} \leq 0.0001$ (one-way ANOVA with Sidak's post hoc test)]. NS indicates no statistically significant difference in $c p x P$-lacZ reporter activity. WT, wildtype. 

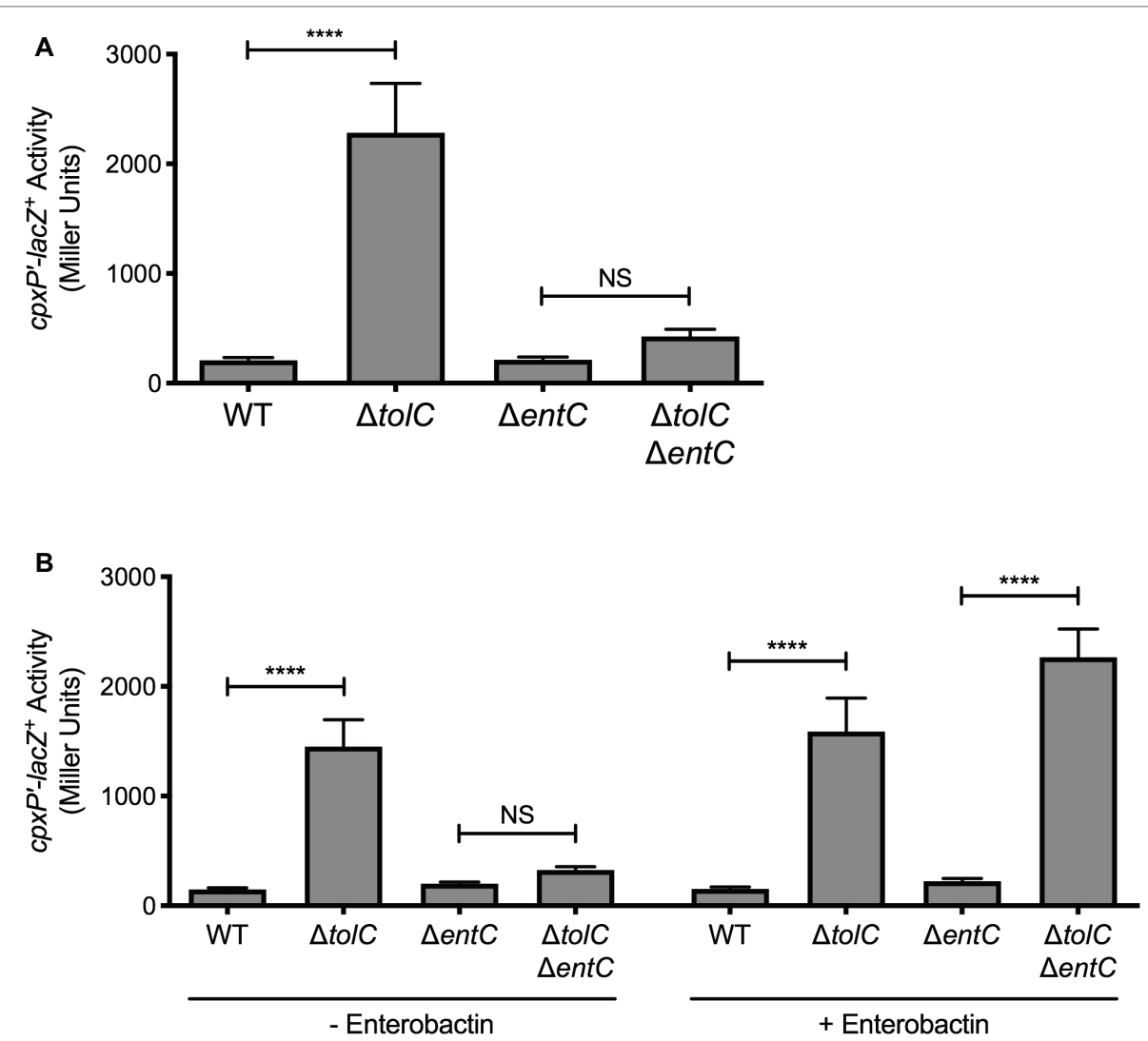

FIGURE 2 | Enterobactin activates the Cpx response in the to/C mutant. (A,B) Expression of the chromosomal cpxP-lacZ transcriptional reporter in wildtype E. coli strain $\mathrm{MC} 4100$, the tolC and entC single mutants, and the to/C entC double mutant. Strains were grown overnight in LB and then subcultured into (A) M9 minimal medium, or (B) M9 minimal medium with (+) or without (-) $10 \mu \mathrm{M}$ enterobactin. As enterobactin is dissolved in $42 \% \mathrm{DMSO}$, an equivalent volume of $42 \%$ DMSO was added to cultures without (-) enterobactin. Bacteria were grown for $20 \mathrm{~h}$ at $37^{\circ} \mathrm{C}$. Cells were lysed with chloroform and SDS, and $\beta$-galactosidase levels were measured after addition of ONPG in a 96-well plate as described in the experimental procedures section. Data show means and standard deviations of three biological replicates. Asterisks indicate a statistically significant difference from the indicated wildtype control ${ }^{\star \star \star \star} p \leq 0.0001$ (one-way ANOVA with Sidak's post hoc test)]. NS indicates no statistically significant difference in cpxP reporter activity. WT, wildtype.

subcultured at a dilution of 1:100 into fresh LB or M9 minimal medium (Difco) and grown for $20 \mathrm{~h}$ at $37^{\circ} \mathrm{C}$ with shaking at 225 RPM. Where indicated, $\mathrm{FeSO}_{4}$ and enterobactin were added to a final concentration of 80 and $10 \mu \mathrm{M}$, respectively. As enterobactin is dissolved in $42 \% \mathrm{DMSO}$, an equivalent volume of $42 \% \mathrm{DMSO}$ was added to the control cultures. $\beta$-galactosidase activity was measured as previously described (Buelow and Raivio, 2005). Bacteria were pelleted by centrifugation at $2880 \times g$ for $10 \mathrm{~min}$. The supernatant was removed, and bacteria were resuspended in $2 \mathrm{ml}$ of $1 \times \mathrm{Z}$ buffer $\left[10 \mathrm{ml}\right.$ of $10 \times \mathrm{Z}$ buffer $\left(600 \mathrm{mM} \mathrm{Na}_{2} \mathrm{HPO}_{4} \cdot 7 \mathrm{H}_{2} \mathrm{O}\right.$, $\left.400 \mathrm{mM} \mathrm{NaH} \mathrm{PO}_{4} \cdot \mathrm{H}_{2} \mathrm{O}, 100 \mathrm{mM} \mathrm{KCl}, 10 \mathrm{mM} \mathrm{MgSO} \cdot \cdot 7 \mathrm{H}_{2} \mathrm{O}\right)$, $90 \mathrm{ml}$ distilled water, $270 \mu \mathrm{l} \beta$-mercaptoethanol]. A volume of $250 \mu \mathrm{l}$ of sample was transferred to a 96-well plate and $\mathrm{OD}_{600}$ was measured using the PerkinElmer Wallac Victor ${ }^{2} 1,420$ plate reader. Chloroform and SDS were used to lyse the remaining cells. A volume of $5 \mu \mathrm{l}$ of sample was added to $195 \mu \mathrm{l}$ of $1 \times$ $\mathrm{Z}$ buffer in a 96-well plate. A volume of $50 \mu \mathrm{l}$ of $10 \mathrm{mg} / \mathrm{ml}$ $o$-nitrophenyl- $\beta$-D-galactopyranoside (ONPG) was added, and hydrolysis of ONPG was measured at an absorbance of $420 \mathrm{~nm}$ $\left(A_{420}\right)$. $A_{420}$ was read 20 times with 45 seconds between each reading.
For Figure 4 and Supplementary Figure S1, bacteria were grown overnight in $\mathrm{LB}$ at $37^{\circ} \mathrm{C}$ with shaking at $225 \mathrm{rpm}$. Bacteria were pelleted by centrifugation at $2880 \times g$ for $10 \mathrm{~min}$, washed once in $1 \mathrm{ml}$ phosphate buffered saline, and resuspended in $2 \mathrm{ml}$ of phosphate buffered saline. Ten microliters of washed bacteria were spotted onto M9 minimal medium agar containing $0.4 \%$ glucose and grown for $24 \mathrm{~h}$ at $37^{\circ} \mathrm{C}$. Bacteria were then scraped off the plate using plastic inoculating loops and resuspended in $2 \mathrm{ml} 1 \times \mathrm{Z}$ buffer. $\beta$-galactosidase activity was measured as described above.

\section{NADH Oxidase Assay}

After growth overnight in $5 \mathrm{ml}$ of LB with shaking at $225 \mathrm{rpm}$, bacteria were diluted by a factor of 1:100 into $5 \mathrm{ml}$ of $\mathrm{M} 9$ minimal medium (Difco) containing $0.4 \%$ glucose and grown for $20 \mathrm{~h}$ at $37^{\circ} \mathrm{C}$. Bacteria were pelleted by centrifugation at $2880 \times \mathrm{g}$ for $10 \mathrm{~min}$ and the pellet was resuspended in $1 \mathrm{ml}$ of cold $50 \mathrm{mM} 4$-morpholineethanesulfonic acid (MES) buffer ( $\mathrm{pH}$ 6.0). Bacteria were pelleted again by centrifugation at $21,130 \times g$ for $1 \mathrm{~min}$. The supernatant was removed and 
the wet weight of the bacteria was determined. Bacteria were resuspended in $1 \mathrm{ml}$ of cold $50 \mathrm{mM}$ MES buffer, $\mathrm{pH}$ 6.0 and $25 \mu \mathrm{l}$ of protease inhibitor cocktail (Sigma-Aldrich) was added for every $100 \mathrm{mg}$ of wet cell weight. Bacteria were then lysed by sonication. Intact cells were removed by centrifugation at $10,000 \times \mathrm{g}$ for $30 \mathrm{~min}$ at $4^{\circ} \mathrm{C}$. A volume of $100 \mu \mathrm{l}$ of sample was added to $890 \mu \mathrm{l}$ of pre-warmed $50 \mathrm{mM}$ MES buffer, $\mathrm{pH} 6.0$ in a 1-ml microrespiration chamber and covered with $150 \mu$ l of light mineral oil to prevent oxygen from dissolving into the medium. The microrespiration chamber was placed in a $30^{\circ} \mathrm{C}$ water bath for $5 \mathrm{~min}$ prior to the addition of $\beta$-NADH. A concentration of $100 \mu \mathrm{M} \beta$-NADH was added and oxygen concentration was measured every $30 \mathrm{~s}$ for 10-15 min using an oxygen MicroOptode sensor (Unisense). Oxygen concentration at each time point was standardized to the oxygen concentration just prior to the addition of $\beta$-NADH. Oxygen consumption for each sample was measured in technical duplicate. The rate of oxygen consumption $\left(\% \mathrm{~min}^{-1}\right)$ was calculated from the linear range of the reaction. The average rate of oxygen consumption of two technical replicates was standardized to the amount of total protein added to the microrespiration chamber. Protein concentration for each sample was determined using the Pierce BCA Protein Assay kit (Thermo Scientific).

\section{Luminescence Assay}

Bacteria were grown overnight in $2 \mathrm{ml}$ of $\mathrm{LB}$ at $30^{\circ} \mathrm{C}$. Bacteria were then diluted at a factor of 1:100 into $2 \mathrm{ml}$ of M9 minimal medium (Difco) containing $0.4 \%$ glucose, $5.34 \mathrm{mM}$ isoleucine, and $6.53 \mathrm{mM}$ valine and grown at $30^{\circ} \mathrm{C}$ with shaking at $225 \mathrm{rpm}$. A volume of $200 \mu \mathrm{l}$ of culture was transferred to a black-walled 96-well microtiter plate $8 \mathrm{~h}$ after subculture and luminescence (in $\mathrm{CPS}$ ) and $\mathrm{OD}_{600}$ were measured as described above. Luminescence [expressed in counts per second (CPS)] and $\mathrm{OD}_{600}$ were read from the microtiter plate for each sample using the PerkinElmer Wallac Victor ${ }^{2} 1,420$ plate reader. entCEBA-lux activity was calculated by subtracting the CPS and $\mathrm{OD}_{600}$ values measured from a blank well containing uncultured $\mathrm{LB}$ from the raw $\mathrm{CPS}$ and $\mathrm{OD}_{600}$ values measured for each sample. The normalized CPS was divided by the normalized $\mathrm{OD}_{600}$ to account for differences in growth between samples.

\section{Statistical Analysis}

Statistical analysis was performed using Prism version 8.2.1 (GraphPad Software). Activity of transcriptional reporters was compared by two-way analysis of variance followed by Sidak's multiple comparison test.

\section{RESULTS}

\section{Iron Limitation Induces the Cpx Response in the to/C Mutant}

To confirm that inhibition of efflux activates the Cpx response in E. coli, we measured Cpx pathway activity in a tolC mutant using a $c p x P$-lacZ transcriptional reporter. No change in $c p x P$-lac $Z$ reporter activity was observed when $E$. coli were grown in LB (Figure 1A). This was surprising given that under similar growth conditions, expression of the periplasmic chaperone Spy has been shown to increase in a tolC mutant via the Cpx response (Rosner and Martin, 2013; Acosta et al., 2014). Nonetheless, when E. coli were grown in M9 minimal medium, we observed a nearly 11 -fold increase in $c p x P$-lacZ activity in the tolC mutant (Figure 1A). This increase was abolished in E. coli lacking $c p x A$ (Figure 1B), suggesting that inhibition of efflux generates envelope stress that is sensed by CpxA. These results suggest that the metabolite(s) responsible for activating the $\mathrm{Cpx}$ response in the $E$. coli tolC mutant is produced in minimal medium, but not in rich medium.

Previous results from our lab have shown that impaired efflux activates the $V$. cholerae Cpx response when iron is limiting (Acosta et al., 2014). As such, we hypothesized that iron may be involved in activation of the Cpx response in the E. coli tolC mutant. As observed previously, mutation of tolC resulted in approximately an 11-fold increase in $c p x P$-lac $Z$ activity when $E$. coli were grown in the iron-depleted M9 minimal medium (Figure 1C). However, when the tolC mutant was grown in M9 minimal medium supplemented with $80 \mu \mathrm{M}$ $\mathrm{FeSO}_{4}$, activation of the Cpx response was no longer observed (Figure 1C). These results suggest that the metabolite(s) responsible for activating the Cpx response in E. coli lacking tolC is produced during iron deprivation.

\section{Enterobactin Activates the Cpx Response in the E. coli tolC Mutant}

Several lines of evidence implicate the siderophore enterobactin in activation of the E. coli Cpx response in the tolC mutant. First, enterobactin is synthesized in response to iron starvation and its production is repressed in the presence of iron by the master iron regulator Fur (Bagg and Neilands, 1985; Brickman et al., 1990). Second, enterobactin is secreted into the environment via TolC (Bleuel et al., 2005) and in the absence of TolC, enterobactin accumulates in the periplasm (Vega and Young, 2014). Finally, enterobactin is structurally similar to vibriobactin (Griffiths et al., 1984), the metabolite responsible for activating the Cpx response in $V$. cholerae efflux mutants (Kunkle et al., 2017). To test whether accumulation of enterobactin is responsible for activating the Cpx response in absence of tolC in $E$. coli, we disrupted enterobactin biosynthesis genetically by deleting entC (Young et al., 1971). Unlike the tolC single mutant where $c p x P$-lac $Z$ activity was increased 11 -fold, there was no increase in $c p x P$-lac $Z$ activity in the tolC entC double mutant (Figure 2A). Furthermore, addition of exogenous enterobactin to the medium restored Cpx pathway activation in the tolC entC double mutant (Figure 2B). Together, these results suggest that enterobactin is responsible for activating the Cpx stress response in the E. coli tolC mutant and demonstrate that accumulation of catechol siderophores generates a Cpx-inducing signal that is conserved in $V$. cholerae and E. coli. 


\section{Impaired Secretion of Enterobactin Decreases NADH Oxidase Activity}

We next sought to determine whether other phenotypes associated with the tolC mutant are due to impaired secretion of enterobactin. While tolC is not essential for growth in rich medium, the growth rate of tolC-deficient $E$. coli is substantially reduced in minimal medium (Dhamdhere and Zgurskaya, 2010; Vega and Young, 2014). One study attributed this phenotype to reduced activity of NADH dehydrogenase of the electron transport chain (Dhamdhere and Zgurskaya, 2010), while another found that the growth defect could be suppressed by the addition of iron (Vega and Young, 2014). Together, these results suggest that enterobactin may reduce $\mathrm{NADH}$ dehydrogenase activity in the tolC mutant. To examine this possibility, we measured $\mathrm{NADH}$ oxidase activity in the tolC and ent $C$ single mutants and tolC ent $C$ double mutant by measuring the rate of oxygen consumption using $\beta-\mathrm{NADH}$ as the electron donor. As expected, oxygen consumption is reduced in the tolC mutant compared to the wildtype (Figure 3). However, oxygen consumption in the tolC entC double mutant is similar to that of the WT and entC single mutant (Figure 3). These results are consistent with the hypothesis that enterobactin is responsible for reduced $\mathrm{NADH}$ dehydrogenase activity in the tolC mutant.

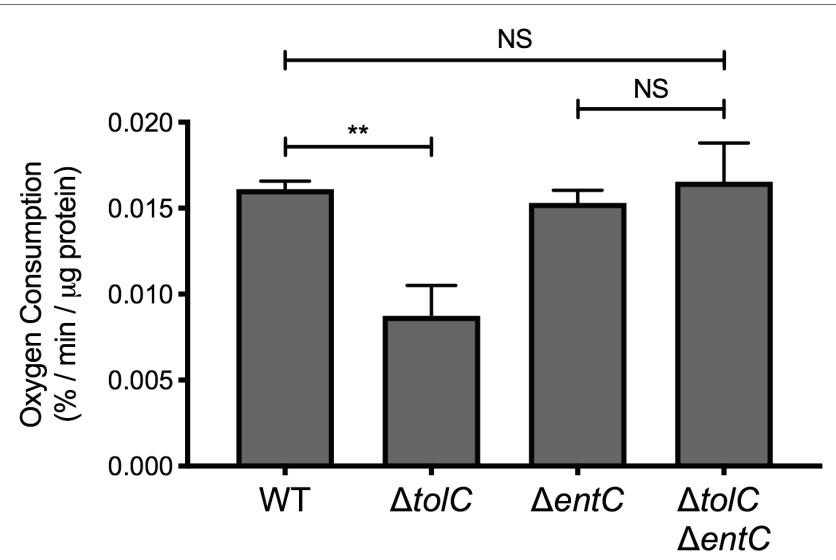

FIGURE 3 | Enterobactin reduces NADH oxidase activity in the tolC mutant. Wildtype (WT) E. coli strain MC4100, the to/C and entC single mutants, and the tolC entC double mutant were subcultured into M9 minimal medium containing $0.4 \%$ glucose after overnight growth in LB medium and grown for $20 \mathrm{~h}$ at $37^{\circ} \mathrm{C}$. Bacteria were collected by centrifugation and washed once in $50 \mathrm{mM}$ MES buffer, pH 6.0. Bacteria were then pelleted by centrifugation, weighed, and resuspended in $1 \mathrm{ml}$ of $50 \mathrm{mM}$ MES buffer, pH 6.0. Bacteria were lysed by sonication. A volume of $100 \mu$ l of cell lysate was diluted in $890 \mu$ of 50 mM MES buffer pre-warmed to $30^{\circ} \mathrm{C}$ in a $1-\mathrm{ml}$ microrespiration chamber. Diluted lysate was covered in light mineral oil to prevent oxygen from dissolving into the system. Oxygen concentration was measured every $30 \mathrm{~s}$ for 10-15 min after the addition of $100 \mu \mathrm{M} \beta-\mathrm{NADH}$ at $30^{\circ} \mathrm{C}$ using an oxygen MicroOptode sensor (Unisense). Oxygen concentration at each time point was standardized to the concentration present just prior to the addition of $\beta-\mathrm{NADH}$. The rate of oxygen concentration per $\mu \mathrm{g}$ of total protein was calculated as described in the experimental procedures section. Data represent the means and standard deviations of three biological replicates. Asterisks indicate a statistically significant difference from the indicated strain $\left[{ }^{\star *} p \leq 0.01\right.$ (oneway ANOVA with Sidak's post hoc test)]. NS indicates no statistically significant difference in the rate of oxygen consumption.

\section{The Cpx Response in the to/C Mutant Remains Active in the Absence of Aerobic Electron Transport Chain Components}

E. coli encode two NADH dehydrogenase isoenzymes that can oxidize $\beta$-NADH, NADH dehydrogenase I (NDH-I), and NADH dehydrogenase II (NDH-II) (Matsushita et al., 1987). Under aerobic conditions, electrons released from $\beta-\mathrm{NADH}$ by NDH-I or NDH-II are first transferred to ubiquinone and then to a terminal oxidase, such as cytochrome $b_{3}$. Given that activity of the NADH oxidation arm of the aerobic respiratory chain is impaired in the tolC mutant (Figure 3), and that NDH-I and cytochrome $b o_{3}$ contribute to $\mathrm{Cpx}$ pathway activity in enteropathogenic E. coli (Guest et al., 2017), we next asked whether NDH-I, NDH-II, or cytochrome $b_{3}$ is required for activation of the Cpx response in the tolC mutant. To test this hypothesis, we mutated each of these electron transport chain components in E. coli containing or lacking tolC and measured Cpx pathway activity using the $c p x P$-lacZ transcriptional reporter. As many of the mutants grew poorly in minimal glucose broth, we determined $c p x P$ lacZ expression from bacteria grown on M9 minimal agar medium. Deletion of tolC activated the Cpx response under these conditions, evidenced by a 5.5 -fold increase in $c p x P$ lac $Z$ activity in the tolC mutant compared to the wildtype (Figure 4). Furthermore, $c p x P$-lacZ expression was increased to a lesser extent in the tolC entC double mutant than in the tolC single mutant (Supplementary Figure S1), confirming that activation of the $\mathrm{Cpx}$ response in the tolC mutant is dependent on enterobactin under these conditions as well. The Cpx response was activated to a similar extent in the tolC nuoA-N (NADH dehydrogenase I), tolC $n d h$ (NADH dehydrogenase II), and tolC $c y o A-E$ (cytochrome $b o_{3}$ ), double mutants as in the tolC single mutant (Figure 4), suggesting that these complexes are not required for activation of the Cpx response in E. coli lacking tolC.

\section{Regulation of Enterobactin Biosynthesis Genes by the Cpx Response}

Previous microarray experiments performed to identify members of the Cpx regulon in enteropathogenic E. coli (EPEC) and E. coli K-12 strain MC4100 found that expression of several genes involved in enterobactin biosynthesis is decreased upon activation of the Cpx response, including ent $A$, ent $B$, ent $C$, and entE (Raivio et al., 2013). To confirm regulation of the enterobactin biosynthesis genes by the $\mathrm{Cpx}$ response, we constructed luminescent transcriptional reporters of EPEC and MC4100 entCEBA expression. Activity of each lux reporter was analyzed in wildtype EPEC or MC4100, mutants containing the cpxA24 allele that constitutively activates the Cpx response, and in E. coli lacking the Cpx response. Mutational activation of the Cpx response in EPEC resulted in a 4-fold decrease in activity of the EPEC entCEBA-lux reporter (Figure 5A). No change in reporter activity was observed in the EPEC $\triangle c p x R A$ mutant (Figure 5A), suggesting that basal expression of the 


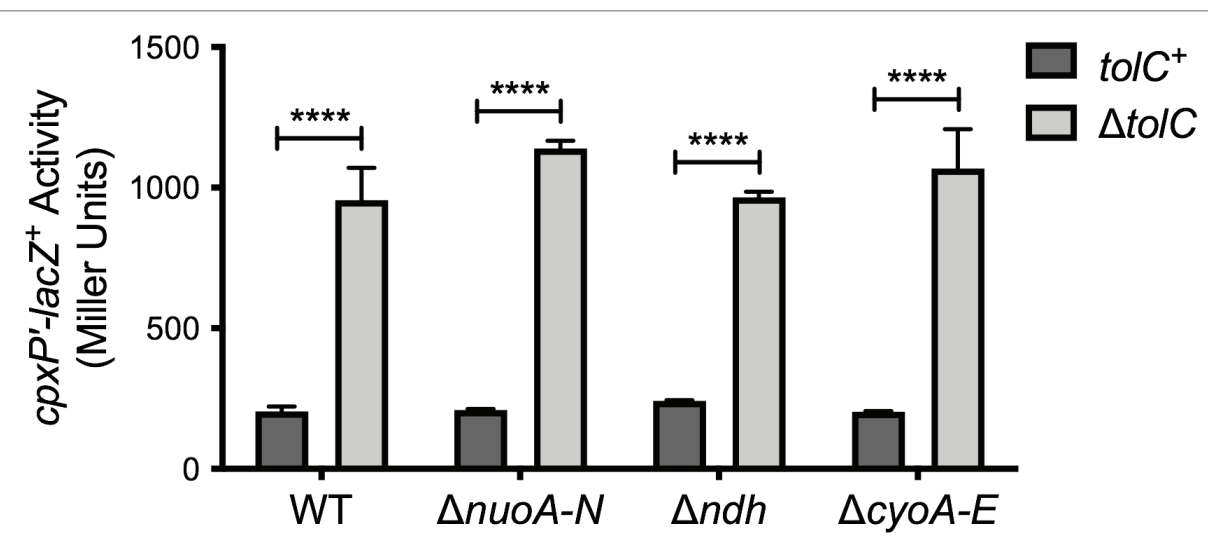

FIGURE 4 | Activation of the Cpx response by deletion of to/C does not require NDH-I, NDH-II, or cytochrome bo ${ }_{3}$. After overnight growth in LB medium, bacteria containing the cpxP-lacZ transcriptional reporter were washed once, and resuspended in phosphate buffered saline. A volume of $10 \mu \mathrm{l}$ of culture was spotted onto M9 minimal medium agar containing $0.4 \%$ glucose and grown at $37^{\circ} \mathrm{C}$ for $24 \mathrm{~h}$. Bacteria were collected using plastic inoculating loops and resuspended in $1 \times Z$ buffer. cpxP-lacZ activity was then measured as described in the experimental procedures section. The strains shown are TR50 and the isogenic $\triangle$ tolC, $\triangle$ nuoABCDEFGHIJKLMN::kan, $\Delta$ ndh::kan, and $\triangle$ cyoABCDE::kan mutants, and the $\triangle$ tolC $\triangle$ nuoABCDEFGHIJKLMN::kan, $\Delta$ tolC $\Delta$ ndh::kan, and $\Delta$ to/C $\triangle c y o A B C D E:: k a n$ double mutants. Data represent the means and standard deviations of three biological replicates. Asterisks indicate a statistically significant difference from the control strain containing a wildtype copy of to/C (to/C+) [**** $\leq \leq 0.0001$ (two-way ANOVA with Sidak's post hoc test)].

enterobactin biosynthesis genes is not affected by loss of the Cpx response. Likewise, activation of the Cpx response in MC4100 led to a 6.2-fold decrease in activity of the MC4100 entCEBA-lux reporter in comparison to the wildtype (Figure 5B). No change in MC4100 entCEBA-lux activity was observed in the MC4100 $c p x R$ mutant (Figure 5B). These data suggest that the transcription of genes involved in enterobactin biosynthesis is repressed upon activation of the Cpx response in EPEC and MC4100.

Intriguingly, we observed differences in basal entCEBA expression between MC4100 and EPEC. Activity of the native entCEBA-lux reporter in MC4100 was 5.1-fold higher than activity of the native entCEBA-lux reporter in EPEC (Supplementary Figure S2A). The promoter regions of the entCEBA operon are substantially different between MC4100 and EPEC (Supplementary Figure S2B). In addition to several base pair substitutions, there is an 186-bp deletion in the promoter region of the EPEC entCEBA operon. There are two possible explanations for the difference in the basal levels of entCEBA transcription in MC4100 and EPEC. The first possibility is that the differences in the DNA sequence of the EPEC entCEBA promoter decrease basal transcription of the entCEBA operon. If true, we would expect that activity of the EPEC entCEBA-lux reporter would decrease in MC4100 and that activity of the MC4100 entCEBA-lux reporter would increase in EPEC. The second possibility is that activity of transcription factors that regulate entCEBA transcription is different between EPEC and MC4100. Here, expression of the EPEC and MC4100 entCEBA-lux reporters would both be similar in EPEC. Likewise, expression of both reporters would be similar in MC4100. We found that activity of the EPEC and MC4100 entCEBA-lux reporters was similar in EPEC, suggesting that the difference in entCEBA transcription is not due to differences in the DNA sequence of the entCEBA promoter regions
(Supplementary Figure S2A). Furthermore, we found that activity of the EPEC entCEBA-lux reporter in MC4100 is actually increased approximately 2 -fold in comparison to the activity of the MC4100 entCEBA-lux reporter in MC4100 (Supplementary Figure S2A). Accordingly, these results suggest that expression of the entCEBA operon is decreased in EPEC through changes in activity of transcriptional regulators.

\section{DISCUSSION}

Multidrug efflux pumps export a wide range of antimicrobial compounds and thus play a major role in resistance of gramnegative bacteria to various antibiotics. However, several studies have revealed that multidrug efflux pumps are involved in cellular processes beyond antibiotic resistance, including cell division, biofilm formation, pathogenesis, cell communication, oxidative and nitrosative stress resistance, and envelope biogenesis (Baranova, 2016; Fahmy et al., 2016; Poole and Fruci, 2016). It has been proposed that drug efflux pumps function to secrete toxic endogenous metabolites that disrupt cellular integrity (Helling et al., 2002; Rosner and Martin, 2009). In this study, we report that the catechol siderophore enterobactin is responsible for activating the Cpx envelope stress response in E. coli lacking TolC, the outer membrane channel of several multidrug efflux systems (Figure 6). While the mechanism by which impaired secretion of enterobactin activates the Cpx response remains to be determined, our data suggest that the respiratory complexes $\mathrm{NDH}-\mathrm{I}, \mathrm{NDH}-\mathrm{II}$, and cytochrome $b o_{3}$ are not required.

The enterobactin secretion pathway begins in the cytoplasm, where enterobactin is synthesized (reviewed in Raymond et al., 2003). Cytoplasmic enterobactin is then transported to the periplasm via the singlet efflux pump EntS (Furrer 

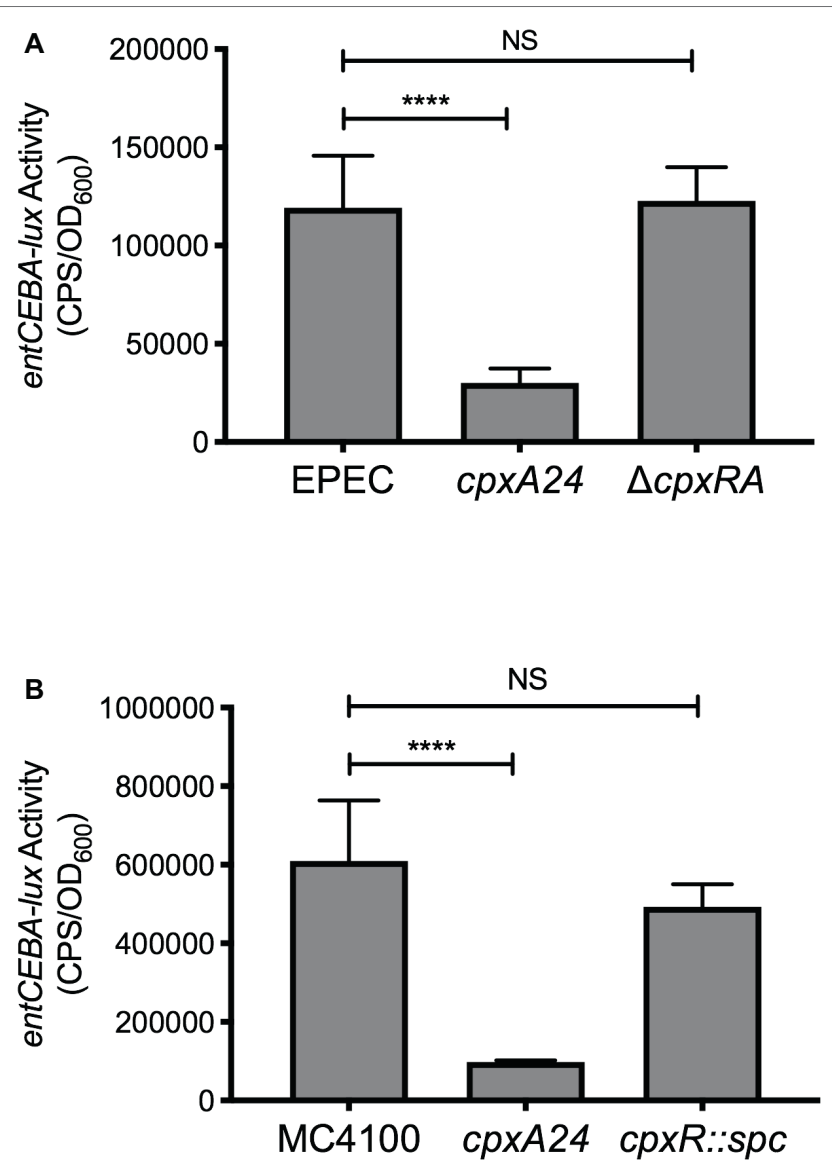

FIGURE 5 | Transcription of the entCEBA operon is repressed by the Cpx response. Bacteria were grown overnight in $\mathrm{LB}$ at $30^{\circ} \mathrm{C}$ with shaking. The following day, bacteria were subcultured into M9 minimal medium supplemented with $0.4 \%$ glucose, $5.34 \mathrm{mM}$ isoleucine, and $6.53 \mathrm{mM}$ valine and grown for $8 \mathrm{~h}$ at $30^{\circ} \mathrm{C}$ with shaking. A volume of $200 \mu$ l of culture was transferred to a black-wall 96-well plate and luminescence [expressed in counts per second (CPS)] and $\mathrm{OD}_{600}$ were read, and entCEBA-lux activity was calculated as described in the experimental procedures section. (A) Activity of the EPEC entCEBA-lux reporter in wildtype EPEC and EPEC containing the cpxA24 or $\triangle c p x R A$ mutation. (B) Activity of the MC4100 entCEBA-lux reporter in wildtype MC4100 and MC4100 containing the cpxA24 or cpxR::spc mutation. Asterisks indicate a statistically significant difference between the indicated strains [ ${ }^{\star * * *} p \leq 0.0001$ (one-way ANOVA with Sidak's post hoc test)]. NS indicates no statistically significant difference in entCEBAlux reporter activity.

et al., 2002). Once in the periplasm, enterobactin is secreted into the environment by one of several TolC-dependent tripartite efflux systems (Bleuel et al., 2005). Enterobactin that has bound to iron is brought back into the cell through the TonB-dependent outer membrane channel FepA, translocated across the periplasm by FepB, and moved into the cytoplasm by the FepCDG inner membrane transporter (reviewed in Guerinot, 1994). Next, the iron-enterobactin complex is hydrolyzed by Fes, which liberates the bound iron and breaks down enterobactin into mono- di- and/or tri-dihydroxybenzoylserine (O’Brien et al., 1971; Brickman and McIntosh, 1992). While our data suggest that impaired secretion of enterobactin induces the Cpx response, the point in the impaired secretion pathway at which envelope stress is generated is unknown. As deletion of tolC does not activate the Cpx pathway in E. coli lacking CpxA, the stress that activates the Cpx response is likely located in the periplasm or inner membrane. In this regard, the toxic defects associated with the $E$. coli tolC mutant grown under iron-limiting conditions are due to the accumulation of periplasmic enterobactin (Vega and Young, 2014). Notably, basal levels of enterobactin in E. coli containing a functional TolC protein do not affect activity of the Cpx response, as cpxP-lac Z expression was unchanged in the ent $C$ single mutant compared to wildtype (Figure 2A). As such, we hypothesize that it is the aberrant accumulation of periplasmic enterobactin in $E$. coli lacking tolC that activates the Cpx response.

Whether impaired secretion of enterobactin is responsible for the pleiotropic phenotypes displayed by bacteria lacking tolC remains to be determined. At present, it is known that enterobactin induces the growth and morphological defects associated with tolC mutants grown in iron-limited medium, but is not required for antibiotic hypersensitivity (Vega and Young, 2014). In a previous study, deletion of tolC in E. coli was found to decrease $\mathrm{NADH}$ oxidase activity when cells were grown in minimal medium, but not rich medium (Dhamdhere and Zgurskaya, 2010). Here, we show that this is due to impaired secretion of enterobactin. There are two possible explanations as to why enterobactin may reduce NADH oxidase activity in the tolC mutant. The first possibility is that enterobactin damages one or both of the NADH dehydrogenase protein complexes, thus reducing their ability to oxidize $\mathrm{NADH}$. The second possibility is that activation of the Cpx response, or another regulatory system, by deleting tolC reduces expression of NDH-I or NDH-II. In support of this hypothesis, CpxR has been shown to directly repress the transcription of the operon encoding NDH-I (Guest et al., 2017). At this point, we are unable to distinguish between these two possibilities.

Although we and others have determined that siderophore accumulation is responsible for activating the Cpx response in the tolC mutant, the nature of the Cpx-inducing stress that is generated under this condition is unknown. Given that activation of the $\mathrm{Cpx}$ response in the $V$. cholerae tolC mutant can be prevented by growing cells anaerobically or by disrupting succinate dehydrogenase, it has been proposed that siderophores chelate iron from the iron-containing cofactors present in aerobic respiratory complexes (Kunkle et al., 2017). Damaged respiratory complexes may either activate the Cpx response directly or increase formation of reactive oxygen species that then generate a Cpx-inducing signal (Kunkle et al., 2017). Here, we found that NDH-I, NDH-II, and cytochrome $b_{3}$ are not individually required for activation of the Cpx response in the E. coli tolC mutant. We were unable to accurately assess the role of succinate dehydrogenase in the activation of the Cpx response in E. coli lacking tolC as the succinate dehydrogenase mutant grew very poorly in 


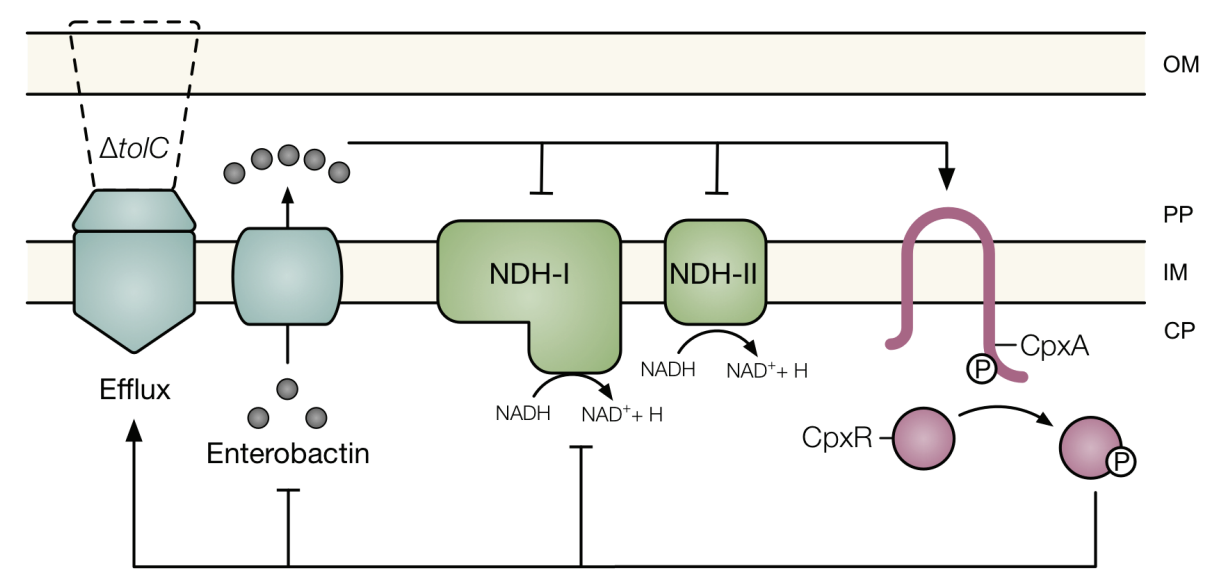

FIGURE 6 | Model of the association between impaired efflux and the Cpx envelope stress response. Under conditions in which efflux through TolC is compromised, the siderophore enterobactin accumulates within the periplasm of $E$. coli. Impaired secretion of enterobactin activates the Cpx response and disrupts $\mathrm{NADH}$ oxidase activity. Cpx regulation of multidrug efflux pumps, respiratory complexes, and enterobactin biosynthesis may provide an adaptive response to enterobactin accumulation. OM, outer membrane; PP, periplasm; IM, inner membrane; CP, cytoplasm; Q, quinone; P, phosphate.

iron-limiting minimal medium (data not shown). Together, these results suggest that succinate dehydrogenase, but not other components of the aerobic electron transport chain, is responsible for activation of the $\mathrm{Cpx}$ response in bacteria lacking tolC. However, we cannot exclude the possibility that respiratory complexes with similar activities to NDH-I, NDH-II, and cytochrome $b_{3}$ function in their absence to induce the Cpx response in the tolC mutant. This would suggest that some aspect related to electron transport chain activity induces the $\mathrm{Cpx}$ response rather than the respiratory complexes themselves.

In agreement with previous transcriptomic data, we found that the Cpx stress response represses expression of the genes for enterobactin biosynthesis in EPEC and E. coli K-12. We also found that basal transcription of the entCEBA operon is decreased in EPEC in comparison to MC4100, likely due to changes in activity of transcription factors in EPEC. As expression of the enterobactin biosynthesis genes is regulated in response to intracellular iron concentrations (Bagg and Neilands, 1985; Brickman et al., 1990), it is possible that intracellular iron concentrations are different in EPEC and MC4100. Alternatively, it is possible that pathogens such as EPEC more tightly control regulation of iron metabolism to facilitate host colonization.

In addition to regulation of enterobactin biosynthesis, other processes regulated by the $\mathrm{Cpx}$ response could facilitate adaptation to the stress caused by enterobactin accumulation. Activation of the Cpx response increases expression of multidrug efflux pumps in E. coli, V. cholerae, Pseudomonas aeruginosa, and Klebsilla pneumoniae (Tian et al., 2016; Guest and Raivio, 2016b), which could lead to increased efflux of periplasmic enterobactin. Indeed, the Cpx-regulated expression of the VexGH efflux pump, which is required for vibriobactin secretion, supports this hypothesis (Kunkle et al., 2017). Furthermore, the Cpx response in E. coli represses expression of several components of the electron transport chain, including succinate dehydrogenase (Raivio et al., 2013; Guest et al., 2017). As such, activation of the Cpx response would decrease expression of the potential target of enterobactin-mediated stress. Through decreased enterobactin biogenesis, increased efflux, and decreased expression of the target respiratory complex, activation of the $\mathrm{Cpx}$ response could mount an effective adaptive response to the stress exerted by enterobactin accumulation (Figure 6).

Several noxious compounds secreted by TolC are present in the host environment. Enteric bacteria such as E. coli and $V$. cholerae are exposed to host-produced factors such as bile and cationic antimicrobial peptides, as well as antibiotics produced by competing members of the intestinal microbiome. Furthermore, E. coli and $V$. cholerae likely synthesize and secrete siderophores in response to the ironpoor environment within the host. As a large number of noxious compounds present in vivo require TolC for secretion, it is possible that they accumulate within the cell faster than can be effluxed through TolC. Activation of the Cpx response could provide protection against the surges in periplasmic siderophore concentrations that occur under these conditions.

\section{DATA AVAILABILITY STATEMENT}

All datasets generated for this study are included in the article/Supplementary Material.

\section{AUTHOR CONTRIBUTIONS}

RG was involved in the design of the study, the acquisition, analysis, and interpretation of the data, and the writing of 
the manuscript. EC, JW, and KS were involved in the acquisition and analysis of the data. TR was involved in the conception and design of the study as well as the writing of the manuscript.

\section{FUNDING}

This work was funded by operating grants MOP 342982 from the Canadian Institutes of Health Research and discovery grant RGPIN 238422-2013 from the Natural Sciences and Engineering Research Council awarded to TR.

\section{REFERENCES}

Acosta, N., Pukatzki, S., and Raivio, T. L. (2014). The vibrio cholerae Cpx envelope stress response senses and mediates adaptation to low iron. J. Bacteriol. 197, 262-276. doi: 10.1128/JB.01957-14

Acosta, N., Pukatzki, S., and Raivio, T. L. (2015). The Cpx system regulates virulence gene expression in vibrio cholerae. Infect. Immun. 83, 2396-2408. doi: 10.1128/IAI.03056-14

Baba, T., Ara, T., Hasegawa, M., Takai, Y., Okumura, Y., Baba, M., et al. (2006). Construction of Escherichia coli K-12 in-frame, single-gene knockout mutants: the Keio collection. Mol. Syst. Biol. 2:2006.0008. doi: 10.1038/msb4100050

Bagg, A., and Neilands, J. B. (1985). Mapping of a mutation affecting regulation of iron uptake systems in Escherichia coli K-12. J. Bacteriol. 161, 450-453.

Baranova, N. (2016). "Involvement of antimicrobial drug efflux systems in bacterial fitness and virulence" in Efflux-mediated antimicrobial resistance in bacteria: Mechanisms, regulation and clinical implications. eds. X. Z. Li, C. A. Elkins, and H. I. Zgurskaya (Cham: Springer International Publishing), 701-727.

Bleuel, C., Grosse, C., Taudte, N., Scherer, J., Wesenberg, D., Krauss, G. J., et al. (2005). TolC is involved in enterobactin efflux across the outer membrane of Escherichia coli. J. Bacteriol. 187, 6701-6707. doi: 10.1128/JB.187.19.6701-6707.2005

Brickman, T. J., and McIntosh, M. A. (1992). Overexpression and purification of ferric enterobactin esterase from Escherichia coli. Demonstration of enzymatic hydrolysis of enterobactin and its iron complex. J. Biol. Chem. 267, 12350-12355.

Brickman, T. J., Ozenberger, B. A., and McIntosh, M. A. (1990). Regulation of divergent transcription from the iron-responsive fepB-entC promoteroperator regions in Escherichia coli. J. Mol. Biol. 212, 669-682. doi: 10.1016/ 0022-2836(90)90229-F

Buelow, D. R., and Raivio, T. L. (2005). Cpx signal transduction is influenced by a conserved N-terminal domain in the novel inhibitor CpxP and the periplasmic protease DegP. J. Bacteriol. 187, 6622-6630. doi: 10.1128/ JB.187.19.6622-6630.2005

Danese, P. N., and Silhavy, T. J. (1997). The sigma(E) and the Cpx signal transduction systems control the synthesis of periplasmic protein-folding enzymes in Escherichia coli. Genes Dev. 11, 1183-1193. doi: 10.1101/gad.11.9.1183

Danese, P. N., and Silhavy, T. J. (1998). CpxP, a stress-combative member of the Cpx regulon. J. Bacteriol. 180, 831-839.

Danese, P. N., Snyder, W. B., Cosma, C. L., Davis, L. J., and Silhavy, T. J. (1995). The Cpx two-component signal transduction pathway of Escherichia coli regulates transcription of the gene specifying the stress-inducible periplasmic protease, DegP. Genes Dev. 9, 387-398. doi: 10.1101/gad.9.4.387

Dhamdhere, G., and Zgurskaya, H. I. (2010). Metabolic shutdown in Escherichia coli cells lacking the outer membrane channel TolC. Mol. Microbiol. 77, 743-754. doi: 10.1111/j.1365-2958.2010.07245.x

Dong, J., Iuchi, S., Kwan, H. S., Lu, Z., and Lin, E. C. (1993). The deduced amino-acid sequence of the cloned cpxR gene suggests the protein is the cognate regulator for the membrane sensor, CpxA, in a two-component signal transduction system of Escherichia coli. Gene 136, 227-230. doi: 10.1016/0378-1119(93)90469-j

Dorel, C., Vidal, O., Prigent-Combaret, C., Vallet, I., and Lejeune, P. (1999). Involvement of the Cpx signal transduction pathway of E. coli in biofilm formation. FEMS Microbiol. Lett. 178, 169-175. doi: 10.1111/j.1574-6968.1999.tb13774.x

\section{ACKNOWLEDGMENTS}

We thank Bernard Lemire for help with designing the NADH oxidase assay. We are thankful to the members of the Raivio lab for their comments. A version of this work has been published as part of the PhD thesis of Guest (2017).

\section{SUPPLEMENTARY MATERIAL}

The Supplementary Material for this article can be found online at: https://www.frontiersin.org/articles/10.3389/fmicb.2019.02776/ full\#supplementary-material

Fahmy, A., Srinivasan, A., and Webber, M. A. (2016). “The relationship between bacterial multidrug efflux pumps and biofilm formation” in Efflux-mediated antimicrobial resistance in bacteria: Mechanisms, regulation and clinical implications. eds. X. Z. Li, C. A. Elkins, and H. I. Zgurskaya (Cham: Springer International Publishing), 651-663.

Furrer, J. L., Sanders, D. N., Hook-Barnard, I. G., and McIntosh, M. A. (2002) Export of the siderophore enterobactin in Escherichia coli: involvement of a $43 \mathrm{kDa}$ membrane exporter. Mol. Microbiol. 44, 1225-1234. doi: 10.1046/j. 1365-2958.2002.02885.x

Griffiths, G. L., Sigel, S. P., Payne, S. M., and Neilands, J. B. (1984). Vibriobactin, a siderophore from vibrio cholerae. J. Biol. Chem. 259, 383-385.

Guerinot, M. L. (1994). Microbial iron transport. Annu. Rev. Microbiol. 48, 743-772. doi: 10.1146/annurev.mi.48.100194.003523

Guest, R. L. (2017). Regulation of respiration by the Cpx response in enteropathogenic Escherichia coli. dissertation/PhD thesis. Edmonton (AB): University of Alberta.

Guest, R. L., and Raivio, T. L. (2016a). Role of the gram-negative envelope stress response in the presence of antimicrobial agents. Trends Microbiol. 24, 377-390. doi: 10.1016/j.tim.2016.03.001

Guest, R. L., and Raivio, T. L. (2016b). "The Cpx inner membrane stress response" in Stress and environmental regulation of gene expression and adaptation in bacteria. ed. F. J. Bruijn (Hoboken, New Jersey, USA: John Wiley \& Sons, Inc.), 1015-1024.

Guest, R. L., Wang, J., Wong, J. L., and Raivio, T. L. (2017). A bacterial stress response regulates expression of respiratory protein complexes to control envelope stress adaptation. J. Bacteriol. 199:e00153-17. doi: 10.1128/JB.00153-17

Helling, R. B., Janes, B. K., Kimball, H., Tran, T., Bundesmann, M., Check, P., et al. (2002). Toxic waste disposal in Escherichia coli. J. Bacteriol. 184, 3699-3703. doi: 10.1128/JB.184.13.3699-3703.2002

Hernday, A. D., Braaten, B. A., Broitman-Maduro, G., Engelberts, P., and Low, D. A. (2004). Regulation of the pap epigenetic switch by CpxAR: phosphorylated CpxR inhibits transition to the phase ON state by competition with Lrp. Mol. Cell 16, 537-547. doi: 10.1016/j.molcel.2004.10.020

Hoang, T. T., Karkhoff-Schweizer, R. R., Kutchma, A. J., and Schweizer, H. P. (1998). A broad-host-range Flp-FRT recombination system for site-specific excision of chromosomally-located DNA sequences: application for isolation of unmarked Pseudomonas aeruginosa mutants. Gene 212, 77-86. doi: $10.1016 / \mathrm{s} 0378-1119(98) 00130-9$

Horiyama, T., and Nishino, K. (2014). AcrB, AcrD, and MdtABC multidrug efflux systems are involved in enterobactin export in Escherichia coli. PLoS One 9:e108642. doi: 10.1371/journal.pone.0108642

Kunkle, D. E., Bina, X. R., and Bina, J. E. (2017). The vibrio cholerae VexGH RND efflux system maintains cellular homeostasis by effluxing vibriobactin. MBio 8, e00126-e00117. doi: 10.1128/mBio.00126-17

Lee, A., Mao, W., Warren, M. S., Mistry, A., Hoshino, K., Okumura, R., et al. (2000). Interplay between efflux pumps may provide either additive or multiplicative effects on drug resistance. J. Bacteriol. 182, 3142-3150. doi: 10.1128/JB.182.11.3142-3150.2000

Li, X.-Z., Plésiat, P., and Nikaido, H. (2015). The challenge of efflux-mediated antibiotic resistance in gram-negative bacteria. Clin. Microbiol. Rev. 28 337-418, doi: 10.1128/CMR.00117-14 
MacRitchie, D. M., Ward, J. D., Nevesinjac, A. Z., and Raivio, T. L. (2008). Activation of the Cpx envelope stress response down-regulates expression of several locus of enterocyte effacement-encoded genes in enteropathogenic Escherichia coli. Infect. Immun. 76, 1465-1475. doi: 10.1128/IAI.01265-07

Matsushita, K., Ohnishi, T., and Kaback, H. R. (1987). NADH-ubiquinone oxidoreductases of the Escherichia coli aerobic respiratory chain. Biochemistry 26, 7732-7737.

McEwen, J., and Silverman, P. (1980). Chromosomal mutations of Escherichia coli that alter expression of conjugative plasmid functions. Proc. Natl. Acad. Sci. USA 77, 513-517.

O’Brien, I. G., Cox, G. B., and Gibson, F. (1971). Enterochelin hydrolysis and iron metabolism in Escherichia coli. Biochim. Biophys. Acta 237, 537-549. doi: 10.1016/0304-4165(71)90274-1

Pogliano, J., Lynch, A. S., Belin, D., Lin, E. C., and Beckwith, J. (1997). Regulation of Escherichia coli cell envelope proteins involved in protein folding and degradation by the Cpx two-component system. Genes Dev. 11, 1169-1182. doi: 10.1101 gad.11.9.1169

Poole, K., and Fruci, M. (2016). "Antimicrobial drug efflux systems as components of bacterial stress responses" in Efflux-mediated antimicrobial resistance in bacteria: Mechanisms, regulation and clinical implications. eds. X. Z. Li, C. A. Elkins, and H. I. Zgurskaya (Cham: Springer International Publishing), 665-700.

Price, N. L., and Raivio, T. L. (2009). Characterization of the Cpx regulon in Escherichia coli strain MC4100. J. Bacteriol. 191, 1798-1815. doi: 10.1128/ JB.00798-08

Raivio, T. L. (2014). Everything old is new again: an update on current research on the Cpx envelope stress response. Biochim. Biophys. Acta 1843, 1529-1541. doi: $10.1016 /$ j.bbamcr.2013.10.018

Raivio, T. L., Laird, M. W., Joly, J. C., and Silhavy, T. J. (2000). Tethering of CpxP to the inner membrane prevents spheroplast induction of the cpx envelope stress response. Mol. Microbiol. 37, 1186-1197. doi: 10.1046/ j.1365-2958.2000.02074.x

Raivio, T. L., Leblanc, S. K. D., and Price, N. L. (2013). The Escherichia coli Cpx envelope stress response regulates genes of diverse function that impact antibiotic resistance and membrane integrity. J. Bacteriol. 195, 2755-2767. doi: $10.1128 /$ JB.00105-13

Raivio, T. L., and Silhavy, T. J. (1997). Transduction of envelope stress in Escherichia coli by the Cpx two-component system. J. Bacteriol. 179, 77247733. doi: $10.1128 /$ jb.179.24.7724-7733.1997

Raymond, K. N., Dertz, E. A., and Kim, S. S. (2003). Enterobactin: an archetype for microbial iron transport. Proc. Natl. Acad. Sci. USA 100, 3584-3588. doi: 10.1073 /pnas. 0630018100

Rinker, S. D., Trombley, M. P., Gu, X., Fortney, K. R., and Bauer, M. E. (2011). Deletion of mtrC in Haemophilus ducreyi increases sensitivity to human antimicrobial peptides and activates the CpxRA Regulon. Infect. Immun. 79, 2324-2334. doi: 10.1128/IAI.01316-10

Rosner, J. L., and Martin, R. G. (2009). An excretory function for the Escherichia coli outer membrane pore TolC: upregulation of marA and soxS transcription and rob activity due to metabolites accumulated in tolC mutants. J. Bacteriol. 191, 5283-5292. doi: 10.1128/JB.00507-09

Rosner, J. L., and Martin, R. G. (2013). Reduction of cellular stress by TolCdependent efflux pumps in Escherichia coli indicated by BaeSR and CpxARP activation of spy in efflux mutants. J. Bacteriol. 195, 1042-1050. doi: 10.1128/ JB.01996-12

Ruiz, C., and Levy, S. B. (2014). Regulation of acrAB expression by cellular metabolites in Escherichia coli. J. Antimicrob. Chemother. 69, 390-399. doi: 10.1093/jac/dkt352

Santos, M. R., Cosme, A. M., Becker, J. D., Medeiros, J. M. C., Mata, M. F., and Moreira, L. M. (2010). Absence of functional TolC protein causes increased stress response gene expression in Sinorhizobium meliloti. BMC Microbiol. 10:180. doi: 10.1186/1471-2180-10-180

Silhavy, T. J., Berman, M. L., and Enquist, L. W. (1984). Experiments with gene fusions. Cold Spring Harbor, NY: Cold Spring Harbor Laboratory.

Silhavy, T. J., Kahne, D., and Walker, S. (2010). The bacterial cell envelope. Cold Spring Harb. Perspect. Biol. 2, a000414-a000414. doi: 10.1101/cshperspect. a000414
Slamti, L., and Waldor, M. K. (2009). Genetic analysis of activation of the vibrio cholerae Cpx pathway. J. Bacteriol. 191, 5044-5056. doi: 10.1128/JB.00406-09

Tal, N., and Schuldiner, S. (2009). A coordinated network of transporters with overlapping specificities provides a robust survival strategy. Proc. Natl. Acad. Sci. USA 106, 9051-9056. doi: 10.1073/pnas.0902400106

Tatsumi, R., and Wachi, M. (2008). TolC-dependent exclusion of porphyrins in Escherichia coli. J. Bacteriol. 190, 6228-6233. doi: 10.1128/JB.00595-08

Taylor, D. L., Bina, X. R., Slamti, L., Waldor, M. K., and Bina, J. E. (2014), Reciprocal regulation of resistance-nodulation-division efflux systems and the Cpx two-component system in vibrio cholerae. Infect. Immun. 82, 2980-2991. doi: 10.1128/IAI.00025-14

Thode, S. K., Rojek, E., Kozlowski, M., Ahmad, R., and Haugen, P. (2018). Distribution of siderophore gene systems on a Vibrionaceae phylogeny: database searches, phylogenetic analyses and evolutionary perspectives. PLoS One 13:e0191860. doi: 10.1371/journal.pone.0191860

Thomason, L., Court, D. L., Bubunenko, M., Costantino, N., Wilson, H. Datta, S., et al. (2007). Recombineering: genetic engineering in bacteria using homologous recombination. Curr. Protoc. Mol. Biol. 78, 1.16.1-1.16.24. doi: $10.1002 / 0471142727 . \mathrm{mb} 0116 \mathrm{~s} 78$

Tian, Z. X., Yi, X. X., Cho, A., O'Gara, F., and Wang, Y. P. (2016). CpxR activates MexAB-OprM efflux pump expression and enhances antibiotic resistance in both laboratory and clinical nalB-type isolates of Pseudomonas aeruginosa. PLoS Pathog. 12:e1005932. doi: 10.1371/journal.ppat.1005932

Vega, D. E., and Young, K. D. (2014). Accumulation of periplasmic enterobactin impairs the growth and morphology of Escherichia coli tolC mutants. Mol. Microbiol. 91, 508-521. doi: 10.1111/mmi.12473

Vogt, S. L., Nevesinjac, A. Z., Humphries, R. M., Donnenberg, M. S., Armstrong, G. D., and Raivio, T. L. (2010). The Cpx envelope stress response both facilitates and inhibits elaboration of the enteropathogenic Escherichia coli bundle-forming pilus. Mol. Microbiol. 76, 1095-1110. doi: 10.1111/ j.1365-2958.2010.07145.x

Vogt, S. L., and Raivio, T. L. (2012). Just scratching the surface: an expanding view of the Cpx envelope stress response. FEMS Microbiol. Lett. 326, 2-11. doi: $10.1111 / j .1574-6968.2011 .02406 . x$

Weber, R. F., and Silverman, P. M. (1988). The cpx proteins of Escherichia coli K12. Structure of the cpxA polypeptide as an inner membrane component. J. Mol. Biol. 203, 467-478. doi: 10.1016/0022-2836(88)90013-7

Wiriyathanawudhiwong, N., Ohtsu, I., Li, Z.-D., Mori, H., and Takagi, H. (2009). The outer membrane TolC is involved in cysteine tolerance and overproduction in Escherichia coli. Appl. Microbiol. Biotechnol. 81, 903-913. doi: 10.1007/ s00253-008-1686-9

Wong, J. L., Vogt, S. L., and Raivio, T. L. (2013). Using reporter genes and the Escherichia coli ASKA overexpression library in screens for regulators of the gram negative envelope stress response. Methods Mol. Biol. 966, 337-357. doi: 10.1007/978-1-62703-245-2_21

Wyckoff, E. E., Smith, S. L., and Payne, S. M. (2001). VibD and VibH are required for late steps in vibriobactin biosynthesis in vibrio cholerae. J. Bacteriol. 183, 1830-1834. doi: 10.1128/JB.183.5.1830-1834.2001

Young, I. G., Langman, L., Luke, R. K., and Gibson, F. (1971). Biosynthesis of the iron-transport compound enterochelin: mutants of Escherichia coli unable to synthesize 2,3-dihydroxybenzoate. J. Bacteriol. 106, 51-57.

Yu, D., Ellis, H. M., Lee, E. C., Jenkins, N. A., Copeland, N. G., and Court, D. L. (2000). An efficient recombination system for chromosome engineering in Escherichia coli. Proc. Natl. Acad. Sci. USA 97, 5978-5983. doi: 10.1073/ pnas. 100127597

Conflict of Interest: The authors declare that the research was conducted in the absence of any commercial or financial relationships that could be construed as a potential conflict of interest.

Copyright (c) 2019 Guest, Court, Waldon, Schock and Raivio. This is an open-access article distributed under the terms of the Creative Commons Attribution License (CC $B Y)$. The use, distribution or reproduction in other forums is permitted, provided the original author(s) and the copyright owner(s) are credited and that the original publication in this journal is cited, in accordance with accepted academic practice. No use, distribution or reproduction is permitted which does not comply with these terms. 